\title{
Philonsorbonne
}

14 | 2020

Année 2019-2020

\section{"Mehr als Gleichnis ». Langage religieux et métaphore chez Wittgenstein et Rosenzweig}

\section{Émeline DURAND}

\section{(2) OpenEdition}

\section{Journals}

Édition électronique

URL : https://journals.openedition.org/philonsorbonne/1552

DOI : 10.4000/philonsorbonne.1552

ISSN : 2270-7336

Éditeur

Publications de la Sorbonne

\section{Édition imprimée}

Date de publication : 14 février 2020

Pagination : 221-235

ISSN : 1255-183X

\section{Référence électronique}

Émeline DURAND, « "Mehr als Gleichnis ». Langage religieux et métaphore chez Wittgenstein et Rosenzweig », Philonsorbonne [En ligne], 14 | 2020, mis en ligne le 08 mars 2020, consulté le 09 juin 2021. URL : http://journals.openedition.org/philonsorbonne/1552 ; DOI : https://doi.org/10.4000/ philonsorbonne. 1552 


\title{
«Mehr als Gleichnis». \\ Langage religieux et métaphore chez Wittgenstein et Rosenzweig
}

\author{
Émeline DURAND
}

Dans un fragment de 1922 intitulé «Von den Gleichnissen ${ }^{1} »$, Kafka demande ce que signifie faire connaître un sens au moyen de paraboles. Celles-ci se révèlent inapplicables à la vie de tous les jours, dans la mesure où elles visent «quelque au-delà légendaire» (irgendein sagenhaftes Drüben), «quelque chose que nous ne connaissons pas », et que l'auteur de la parabole est lui-même incapable de décrire plus précisément, de sorte que la parabole constitue l'unique accès à ce sens qui est le sien. Inutiles à la vie, hermétiques à notre quête de sens, les paraboles semblent alors ne nous enseigner qu'une seule leçon: que ce qui est insaisissable est insaisissable. Pourtant, c'est à tout autre chose qu'à de l'insaisissable que nous appliquons chaque jour nos efforts. Ne reste peut-être qu'à suivre les paraboles, de sorte à devenir nous-mêmes des paraboles, nous libérant ainsi des peines de chaque jour. Mais l'apologue conclut qu'au jeu de la recherche de leur sens, nous sommes toujours perdants, que le jeu et la défaite aient lieu dans la réalité (in Wirklichkeit) ou dans la parabole (im Gleichnis).

Par le lien qu'elle établit entre le souci de l'ordinaire, le mythe d'un au-delà posé comme terme prétendu de notre quête, et les formes de langage par lesquelles nous nous efforçons d'en dire quelque chose, l'insondable parabole de Kafka consonne avec les réflexions que mènent, à la même époque, Ludwig Wittgenstein dans le Tractatus logico-

\footnotetext{
1. Franz Kafka, «Des paraboles », Nouvelles et récits, Euvres complètes I, édition publiée sous la direction de Jean-Pierre Lefebvre, avec la collaboration d'Isabelle Kalinowski, Bernard Lortholary et Stéphane Pesnel, Paris, Gallimard, "Bibliothèque de la Pléiade », 2018, p. 923.
} 
philosophicus et Franz Rosenzweig dans L'Étoile de la Rédemption. Une même question retient l'attention des trois auteurs : que peut bien vouloir dire une parabole ou métaphore (Gleichnis) qui n'est l'image de rien?

On doit à Hilary Putnam la première suggestion d'une affinité entre les pensées de Ludwig Wittgenstein et de Franz Rosenzweig, un rapprochement discuté par la suite par Cora Diamond et Paul Franks'. Si des similarités biographiques ne sont pas suffisantes à fonder une proximité de pensée, elles sont toutefois pertinentes pour deux auteurs aux yeux de qui les problèmes de la philosophie purent souvent apparaître comme des problèmes $d u$ philosophe $e^{3}$. Rappelons donc l'épreuve matérielle et spirituelle que fut pour Rosenzweig et Wittgenstein l'expérience de la Grande guerre; la rédaction, au lendemain de celle-ci, d'une œuvre radicalement singulière, venant donner du front contre les limites de la philosophie ; enfin la décision, après cette première publication, de se retirer de la philosophie pour entrer « dans la vie ». Ce rapprochement ne doit pas occulter tout ce qui sépare les deux penseurs, en particulier quant au rôle de la religion dans leur parcours intellectuel. Ce qui n'est chez Wittgenstein qu'un "point de vue religieux » dont l'interprétation fait encore débat ${ }^{4}$ constitue le cœur de la pensée de Rosenzweig, dont l'œuvre articule une réflexion philosophique nourrie de l'histoire de la pensée allemande et une perspective théologico-religieuse ancrée dans les sources juives. Si tous deux prennent position contre la manière traditionnelle de poser et de résoudre les problèmes philosophiques, en s'appuyant sur une philosophie conçue comme critique du langage «mais pas au sens de Mauthner ${ }^{5}$; si dans les deux cas la méthode adoptée a pu se qualifier de «grammaticale ${ }^{6}$, le langage qui sert de référence

2. Voir Hilary Putnam, Jewish Philosophy as a Guide to Life. Rosenzweig, Buber, Levinas, Wittgenstein, Bloomington and Indianapolis, Indiana University Press, 2008 (La Philosophie juive comme guide de vie, trad. Anne Le Goff, Paris, Éditions du Cerf, 2011) ; Cora Diamond, "Wittgenstein on Religious Belief: The Gulfs Between Us », in Religion and Wittgenstein's Legacy, D.Z. Phillips, Mario von der Ruhr (éds.), Aldershot/Burlington, Ashgate, 2005, p. 99-137 ; Paul Franks, «Talking of Eyebrows: Religion and the Space of Reasons after Wittgenstein, Rosenzweig and Diamond », ibidem, p. 139-159.

3. Franz Rosenzweig, "Das neue Denken», Der Mensch und sein Werk: Gesammelte Schriften, III: Zweistromland. Kleinere Schriften zu Glauben und zu Denken, hrsg. von Reinhold und Annemarie Mayer, La Haye, M. Nijhoff, 1982, p. 160 ; « La pensée nouvelle », Foi et savoir. Autour de L'Étoile de la Rédemption, introduit, traduit et annoté par G. Bensussan, M. Crépon et M. de Launay, Paris, Vrin, 2001, p. 168.

4. Voir Norman Malcolm, Wittgenstein : A Religious Point of View ?, London, Routledge, 1993 ; Wittgenstein, un point de vue religieux ?, traduit et postfacé par Michel Le Du, Paris, Éditions de l'Éclat, 2014.

5. Ludwig Wittgenstein, Tractatus logico-philosophicus, 4.0031, trad. fr. Gilles-Gaston Granger, Paris, Gallimard/Tel, 1993, p. 51. Mauthner est évoqué en termes critiques dans la correspondance de Rosenzweig, bien que celui-ci reconnaisse également la proximité de leur rapport au langage. Voir Franz Rosenzweig, Die "Gritli"-Briefe: Briefe an Margrit Rosenstock-Huessy, hrsg. von Inken Rühle und Reinhold Mayer, mit einem Vorwort von Rafael Rosenzweig, Tübingen, Bilam Verlag, 2002, p. 765-766.

6. F. Rosenzweig, «Das neue Denken», op. cit., p. 151 ; «La pensée nouvelle », op. cit., p. 161 . 
à Rosenzweig est avant tout le texte de la Bible hébraïque, et non les usages de la langue courante comme chez le second Wittgenstein.

Ces points de convergence et ces différences se retrouvent dans la manière dont Rosenzweig et Wittgenstein ont approché le problème du langage religieux. Cette expression propre à la philosophie contemporaine de la religion, traduction de l'anglais religious language, renvoie à tout ce que nous disons sur Dieu. Plus que sur les contenus du dogme, avec lesquels l'un et l'autre penseur entretiennent un rapport tout à fait distancié, c'est donc sur les énoncés attribués au sujet croyant, les formes dans lesquelles l'expérience religieuse se dit, que porte leur interrogation. Pareils énoncés soulèvent la question de l'adéquation de notre langage à la tâche que la religion et la théologie lui confient: comment la parole humaine, en sa finitude, pourrait-elle exprimer la transcendance du divin ? Sans doute ne peut-elle satisfaire à cette tâche qu'à condition d'assumer son caractère anthropomorphique : en raison des moyens limités qui sont les nôtres, nous appliquerions à Dieu, à titre métaphorique, des termes tirés du monde sensible et de la sphère des affects humains. La Bible elle-même regorgerait de telles analogies, tout particulièrement dans les livres poétiques que sont les Psaumes ou le Cantique des Cantiques. Le langage religieux serait par essence une expression métaphorique, justifiant la pratique de l'interprétation allégorique, soucieuse de déceler la signification vraie du texte derrière son sens obvie.

Pour Wittgenstein et Rosenzweig, cependant, considérer ces usages du langage comme métaphoriques ne va pas de soi. Leur examen du rôle que joue la métaphore dans les énoncés religieux, entre innovation sémantique et opacité du sens, les conduit à suggérer que le problème du langage religieux n'est peut-être pas tant insoluble que mal formulé, voire dénué de pertinence. L'impossibilité de renoncer à la métaphore pour parler «directement» de Dieu ou de l'expérience religieuse peut toutefois susciter l'impression que nous nous heurtons désespérément aux bornes du langage, de sorte que c'est finalement au non-sens que devrait être reconduite l'expression faussement métaphorique de la parole croyante. En suivant les analyses de l'un et l'autre auteur, nous voudrions montrer qu'une autre lecture est possible, qui en vient à dévoiler, au cœur de leur pensée, la commune reconnaissance d'une propriété inattendue du langage : son caractère miraculeux.

Nous suivrons pour cela la piste qu'offre, dans certains de leurs textes, la méditation sur le mot de Gleichnis. Il s'agira d'abord de montrer que, partant de prémisses différentes, Rosenzweig et Wittgenstein en viennent tous deux à conclure que le langage religieux fait de l'image un usage qui lui est essentiel (I). Une divergence apparaîtra alors quant à la capacité de cette image à faire sens: si, pour Rosenzweig, l'autonomie de la métaphore permet précisément l'accès à la signification, elle semble, chez Wittgenstein, mener droit au non-sens des énoncés éthiques et religieux (II). Pourtant, c'est justement dans cet échec apparent de l'expression linguistique que se loge le miracle de «l'existence du langage», auquel nous tenterons de faire droit au terme de notre analyse (III). 
Il faut rappeler, au préalable, que les deux auteurs se sont intéressés à des énoncés religieux de nature différente. L'Étoile de la Rédemption, le grand œuvre de Franz Rosenzweig publié en 1921, se réfère aux sources juives que sont la Bible hébraïque, la tradition rabbinique et la liturgie synagogale ; sa réflexion porte avant tout sur la Parole révélée de Dieu, non sur ce que les hommes disent de lui. Cependant, tout le propos de L'Étoile est de remettre en perspective cette distinction, en soulignant qu'il existe une unité immédiate entre Menschenwort et Gotteswort : s'il est vrai que « Dieu parle partout dans les paroles de l'homme ${ }^{7}$, lire la Bible ne signifiera rien d'autre que de «chercher la parole de l'homme dans la parole de Dieu ${ }^{8}$. Wittgenstein, pour sa part, s'intéresse aux énoncés que les croyants sont susceptibles de prononcer, dont la Conférence sur l'éthique (1929) et les Leçons sur la croyance religieuse (1938) offrent de nombreux exemples: «Je m'étonne de l'existence du monde», "J'éprouve un sentiment de sécurité absolue », " Je crois au Jugement dernier », " Ma maladie est une punition », «L'œil de Dieu voit tout », «Il se pourrait que nous nous voyions après la mort ». Devant pareils propos, il faut commencer par reconnaître qu'en un certain sens nous n'y comprenons rien; c'est ce qui fait d'eux un véritable objet de l'interrogation philosophique, s'il est vrai que les problèmes philosophiques sont de la forme « Je ne m'y retrouve pas »?.

En première approche, ces propos paraissent explicables par la notion d'anthropomorphisme, qui permettrait de rendre compte de leur caractère métaphorique - le sens a bien fait l'objet d'un déplacement - tout en témoignant des limites intrinsèques de l'expression humaine. Ainsi, dans la plupart des propos religieux, «le mot [Dieu] est utilisé comme un mot représentant une personne. Dieu voit, il récompense, etc. ${ }^{10}$. Ce mot semble même se trouver au cœur d'une «vaste allégorie très élaborée qui Le représente comme un être humain doté de grands pouvoirs dont nous essayons d'obtenir la grâce, etc. ${ }^{11}$. Dans la tradition juive,

7. Franz Rosenzweig, Der Stern der Erlösung, Frankfurt am Main, Suhrkamp, 1988 (rééd. 2015), p. 407 ; L'Étoile de la Rédemption, traduit de l'allemand par Alexandre Derczanski et Jean-Louis Schlegel, nouvelle édition revue et annotée par Jean-Louis Schlegel, Paris, Éditions du Seuil, 2003, p. 508-509.

8. Ibidem, p. 221 (trad. française p. 281).

9. Ludwig Wittgenstein, Recherches philosophiques, § 123, trad. fr. par F. Dastur, M. Élie, J.-L. Gautero, D. Janicaud, É. Rigal, Paris, Gallimard/Tel, 2015, p. 87.

10. Ludwig Wittgenstein, «Lectures on Religious Belief», in Lectures and Conversations on Aesthetics, Psychology and Religious Belief, compiled from Notes taken by Yorick Smythies, Rush Rhees and James Taylor, edited by Cyril Barrett, Oxford, Basil Blackwell, 1967, p. 59.

11. Ludwig Wittgenstein, "Conférence sur l'éthique», in Philosophica III. Conférence sur l'éthique, Remarques sur Le Rameau d'or de Frazer, Cours sur la liberté de la volonté, textes établis par James C. Klagge et Alfred Nordmann, traduits par Jean-Pierre Cometti, Gérard Granel et Élisabeth Rigal, Mauvezin, Éditions T.E.R., 2001, p. 16. 
l'anthropomorphisme de la Bible n'est souvent reconnu que pour être critiqué, par exemple dans le Guide des égarés de Maïmonide ou le Traité théologico-politique de Spinoza. L'explication par l'anthropomorphisme laisse toutefois Rosenzweig et Wittgenstein insatisfaits et les conduit à envisager autrement l'usage métaphorique du langage.

À l'occasion d'une recension des premiers volumes de l'Encyclopaedia Judaica, rédigée en 1928, Rosenzweig réagit sévèrement à la critique traditionnelle des anthropomorphismes. En expliquant cette forme d'expression par les carences du langage, voire par les capacités limitées de la pensée humaine - condamnée à ne pouvoir saisir le spirituel qu'à travers le sensible, le supra-humain qu'à travers l'analogie avec l'humain -, on se laisse aller à une mauvaise théorie de la connaissance, oubliant qu'il n'existe pas d'autre langue, d'autre pensée, d'autre expérience humaines auxquelles nous pourrions nous référer pour qualifier les nôtres de «limitées». Pour comprendre la véritable fonction des anthropomorphismes, il faut les reconduire à leur sol empirique, c'est-à-dire à l' "expérience théologique " dont ils sont l'expression, qui n'est autre qu'une expérience de Dieu (Gotteserfahrung) dans sa rencontre avec l'humain (gottmenschliche Begegnung). Les anthropomorphismes ne font que recueillir le sens de cette rencontre: dépourvus de tout statut iconique, ils n'ont pas vocation à représenter les propriétés de Dieu. Lorsque le Psaume 18 invoque les oreilles ouvertes de Dieu ou ses narines enflammées par la colère, ce n'est pas pour tracer un portrait du divin visage, mais pour montrer que Dieu répond à la façon dont l'homme l'interpelle : "Là où il y a quelque chose à voir, Dieu y jette un œil ; là où un être humain appelle, Dieu ouvre une oreille; là où un être humain se prête à l'écoute ou au contraire se bouche les oreilles, la voix de la bouche divine peut remplir cette oreille; là où il tend la main pour implorer de l'aide, la main de Dieu peut la saisir $[\ldots]^{12} »$. Contrairement à la poésie homérique, qui se tient, comme tout ce qui est grec, sous la loi du visible, la langue de la Bible ne fixe pas l'être dans l'image, mais se confie à la parole pour évoquer des rencontres constamment renouvelées dans le temps. La critique traditionnelle des anthropomorphismes occulte donc ce qui devrait être perçu comme un authentique problème théologique, qui ne consiste pas à savoir comment l'humain parle de Dieu, mais comment il parle avec Dieu dans l'expérience de la rencontre ${ }^{13}$.

12. F. Rosenzweig, «Zur Encyclopaedia Judaica », Gesammelte Schriften III, op. cit., p. 739 ; «À propos de l'Encyclopedia Judaica », L'Écriture, le verbe et autres essais, traduction, préface et notes de Jean-Luc Évard, Paris, Presses Universitaires de France, 1998, p. 41-42.

13. Les anthropomorphismes bibliques et rabbiniques sont au cœur de l'analyse de Paul Franks («Talking of Eyebrows », op. cit., notamment p. 148-155). Franks conclut, à la suite de Cora Diamond, que l'on peut appliquer le concept wittgensteinien d'usage secondaire aux tournures anthropomorphiques du langage religieux telles que Rosenzweig les comprend: "All these 'anthropomorphic' statements, then, may be understood as expressions of encounters between God and man, which determine the application to God in a secondary sense of terms applied to man in a primary sense » (op. cit., p. 151). Cependant, la fin de l'article suggère que l'interprétation de Rosenzweig demeure limitée par son manque de sensibilité à la notion d'usage, ce qui le conduit à rejeter de façon définitive certaines 
On trouve chez Wittgenstein un écho à l'argument selon lequel toute évaluation de la complétude de notre langage est impossible, ou dépourvue de sens ${ }^{14}$. Les Leçons sur la croyance religieuse soulignent également le caractère problématique de l'image dans le langage religieux. Wittgenstein rappelle qu'en contexte chrétien, c'est essentiellement au moyen de représentations picturales que nous apprenons à utiliser les mots de la religion, notamment le mot «Dieu». Cependant, le maniement de ces images suppose une méthode toute différente de celle qui a cours avec les images ordinaires : non seulement je ne puis montrer la chose représentée elle-même, mais il n'existe pas même de technique de comparaison entre l'image et ce qu'elle représente, par exemple entre les fresques de la chapelle Sixtine et le déroulement de la Création ${ }^{15}$. Le même raisonnement vaut des usages "imagés » du langage. L'image est la source de notre incompréhension face aux énoncés religieux, puisque des phrases comme « L'œil de Dieu voit tout » associent un usage particulier de mots ordinaires avec une certaine image. Mais dire que le croyant fait usage d'une certaine image ne revient pas à dévaloriser ce propos, ni à le retraduire en des termes que le croyant refuserait : c'est faire une remarque grammaticale, portant sur l'usage que le croyant fait de cette phrase, usage qu'attestent les conséquences qu'il en tirera dans ses propos ultérieurs et ses actions ${ }^{16}$. On ne saurait donc réduire ces propos à une certaine «attitude » qui pourrait tout aussi bien s'exprimer en d'autres termes : la phrase «Il se pourrait que nous nous voyions après la mort » ne dit pas la même chose que «J'ai beaucoup d'affection pour vous » (au point que je souhaiterais vous revoir après la mort). La phrase dit ce qu'elle dit, et non pas ce que l'on pourrait formuler autrement. Le choix de l'image lui est donc essentiel ; il se pourrait même que «tout le poids réside dans l'image ${ }^{17}$ ». La deuxième partie des Recherches philosophiques illustre ce raisonnement avec l'exemple de l'expression « je l'ai compris avec mon cœur» :

tournures (par exemple les anthropomorphismes homériques ou ceux de la tradition mystique du Shi'ur Qomah); dans une perspective wittgensteinienne, il devrait demeurer possible de concevoir un usage donnant un sens à ces expressions (op. cit., p. 154-155).

14. L. Wittgenstein, Recherches philosophiques, op. cit., § 18, p. 34 : « Si tu souhaites dire qu'ils ne sont pas complets, demande-toi si notre langage est complet; - s'il l'était avant que le symbolisme chimique et la notation infinitésimale lui aient été incorporés ; car ce sont, pour ainsi dire, des faubourgs de notre langage. (Et à partir de combien de maisons ou de rues une ville devient-elle une ville ?) ».

15. L. Wittgenstein, «Lectures on Religious Belief», op. cit., p. 63 : «I could show Moore the pictures of a tropical plant. There is a technique of comparison between picture and plant. If I showed him the picture of Michelangelo and said : "Of course, I can't show you the real thing, only the picture"... The absurdity is, I've never taught him the technique of using this picture $»$.

16. L. Wittgenstein, Recherches philosophiques, op. cit., § 486, p. 196 : «Une conclusion est le passage à une assertion, mais aussi le passage au comportement correspondant à cette assertion. "Je tire les conséquences" non seulement en parole, mais aussi en acte ».

17. L. Wittgenstein, «Lectures on Religious Belief», op. cit., p. 72. De même, dans L'Étoile de la Rédemption, la Gleichnis n'est jamais un accessoire décoratif du dire, mais un élément essentiel de ce qui est dit (Der Stern der Erlösung, op. cit., p. 224 ; trad. française p. 284). 
Mais qu'en est-il d'une expression telle que : "Quand tu l'as dit, je l'ai compris avec mon cœur », prononcée en montrant son cœur de la main ? Ne veut-on rien dire par ce geste ?! Évidemment que si. Ou a-t-on conscience d'employer ici une simple image ? - Certainement pas. - Ce n'est ni une image que nous choisirions, ni une comparaison, et c'est pourtant une expression imagée. [Es ist nicht ein Bild unserer Wahl, nicht ein Gleichnis, und doch ein bildlicher Ausdruck $]^{18}$.

C'est donc par essence, et non par défaut, que le langage religieux a recours à des images dont il ne peut se passer ${ }^{19}$. Comment nommer de telles images? Wittgenstein et Rosenzweig optent tous deux pour le terme de Gleichnis, que les mots français d'analogie, de parabole, d'allégorie, de comparaison et de métaphore rendent imparfaitement. Le terme renvoie d'une part à l'existence d'une relation de similitude ou d'équivalence (gleich) entre deux choses, d'autre part à la figure de style qui, en vertu de cette ressemblance, rapproche ces deux choses l'une de l'autre dans le discours. Dans le chapitre central de L'Étoile de la Rédemption, Rosenzweig fait jouer ces deux sens pour justifier que l'expérience de la Révélation puisse être décrite dans les termes de l'amour humain : parce que l'homme fut créé à l'image et à la ressemblance de Dieu $(G n 1,26)$, il est légitime de porter le regard sur l'existence de la créature pour y découvrir le sens que nous pouvons donner au phénomène de la Révélation. La Gleichnis, à ce titre, n'est pas un simple ornement du style, mais un instrument heuristique, permettant aussi bien de découvrir la signification destinale de l'amour (en Dieu) que de décrire son effet en l'homme (la transformation du Soi muet en âme parlante).

Ce double sens de la ressemblance dans l'être et dans le langage se retrouve dans le Tractatus, où Gleichnis désigne tantôt la ressemblance entre le signe propositionnel et ce qu'il représente (4.012), tantôt la figure de style participant du caractère imagé (Bildhaftigkeit) de notre langage (4.015), tantôt l'analogie à vertu pédagogique (4.063), tantôt enfin une simple image de la vérité, par opposition à la vérité elle-même (5.5563). La Conférence sur l'éthique emploie le terme anglais de simile, que Wittgenstein avait préconisé pour la traduction de la proposition $4.015^{20}$. Une conversation de 1930 avec Waismann confirme que c'est bien Gleichnis que Wittgenstein a en tête dans la Conférence sur l'éthique ${ }^{21}$. Or, dès lors que la Gleichnis est

18. L. Wittgenstein, Recherches philosophiques, op. cit., II-iv, p. 254.

19. Sur le sens de ce caractère essentiel de l'image, voir C. Diamond, «Wittgenstein on Religious Belief: The Gulfs Between Us », op. cit., notamment p. 119-131.

20. L. Wittgenstein, Letters to C.K. Ogden, Oxford/London, Basil Blackwell and Routledge \& Kegan Paul, 1973, p. 26 : "Here instead of likenesses the plural form of "simile" would - I think - be better. This prop[osition] in English seems to me very awkward and unclear but I can't mend it. Would it perhaps be better to put instead of "... of the whole pictorial nature..." "of all the imagery of our language"? ».

21. L. Wittgenstein, Werkausgabe, Bd. 3: Ludwig Wittgenstein und der Wiener Kreis. Gespräche, aufgezeichnet von Friedrich Waismann, aus dem Nachlaß herausgegeben von 
indispensable à la mise en mots de l'expérience religieuse et ne saurait être remplacée par une autre manière de dire, peut-on encore se permettre de voir en elle une « simple » métaphore ?

\section{II}

C'est ce que semblent suggérer les premiers mots de l'analyse rosenzweigienne de la Révélation : «L'amour est fort comme la mort. [...] Comme tout amour humain, ce n'est là qu'une analogie [nur ein Gleichnis $]^{22} »$. La perspective, toutefois, ne tarde pas à se renverser, grâce à « l'analyse grammaticale» à laquelle procède le philosophe conformément à la méthode qui est la sienne dans la partie centrale de L'Étoile de la Rédemption: un déchiffrement du sens des trois événements que sont la Création, la Révélation et la Rédemption à partir des modalités linguistiques propres au texte biblique. Après le récit de l'origine du monde, traduisant l'événement de la Création (Genèse), et avant d'en venir à l'expression chorale qui anticipe l'avènement de la Rédemption (Psaumes), Rosenzweig se penche sur le texte où la parole de l'homme et la parole de Dieu se réunissent en un dialogue, modalité propre de la Révélation. Là où l'on attendrait, pour l'illustrer, une référence aux entretiens de Moïse avec Dieu dans l'Exode, le choix de Rosenzweig se porte alors sur le Cantique des Cantiques, un texte dont la canonicité a longtemps fait débat et dont le caractère profane continue de diviser les interprètes. Faut-il comprendre que notre auteur se rattache à la tradition de l'interprétation allégorique, qui voit dans le duo des fiancés du Cantique une allégorie de la relation entre Dieu et son peuple, entre Dieu ou le Christ et l'âme du croyant, ou encore entre le Christ et l'Église?

Rosenzweig renvoie bien plutôt dos-à-dos l'interprétation allégorique et l'interprétation naturaliste, qui voit dans le Cantique un poème d'amour purement humain. L'une et l'autre ont pour principe d'éliminer la métaphore en tant que telle : l'interprétation naturaliste, en prétendant s'en tenir au sens obvie, nie que le Cantique soit une métaphore («kein Gleichnis»); l'interprétation allégorique, en faisant fi du sens obvie, réduit celui-ci à n'être rien qu'une métaphore ("nur ein Gleichnis »). Or, dans le Cantique, la métaphore n'est pas un simple moyen de présenter la relation de Dieu à l'homme à travers l'amour de la Sulamite et de son bien-aimé : le texte laisse au contraire la métaphore à son autonomie, sans l'accompagner d'aucune explication. La métaphore est à l'œuvre sans référence à cela même dont elle

B.F. McGuinness, Frankfurt am Main, Suhrkamp, 1984, p. 117 : «Die Reden der Religion sind auch kein Gleichnis; denn sonst müßte man es auch in Prosa sagen können ». Voir Wittgenstein et le cercle de Vienne, trad. Gérard Granel, Mauvezin, T.E.R., 1991, p. 91 (traduction modifiée).

22. F. Rosenzweig, Der Stern der Erlösung, op. cit., p. 174 (trad. française p. 224-225). 
devrait être la métaphore, le signifiant sans aucune référence au signifié ${ }^{23}$, et pourtant, il est manifeste qu' « une signification suprasensible y élève [überhöht] une signification sensible ${ }^{24} \gg$. La Gleichnis apparaît alors comme une figure du dépassement du sens sensible vers le sens spirituel, qui ne rompt pourtant pas avec son ancrage dans le sensible. C'est pourquoi elle caractérise ces deux phénomènes "sensibles-suprasensibles" (sinnlichübersinnlich) que sont l'amour et le langage, dont la nature est de renvoyer toujours au-delà d'eux-mêmes. Entre l'homme et son Dieu, les mots d'amour et de langage ne sont donc pas employés à titre simplement métaphorique : ils sont au contraire une possession partagée qui permet la rencontre des voix humaine et divine dans le dialogue. Être en vérité une métaphore (wahrhaft Gleichnis), c'est être plus que métaphore (mehr als Gleichnis ${ }^{25}$ ).

La leçon du Cantique est alors la suivante: dans l'ordre linguistique, éminemment dans le texte biblique, la signification n'est pas à rechercher dans la substitution du spirituel au sensible, mais dans leur conjonction au sein d'un même symbole. Toute parole, comme tout amour véritable, se donne à déchiffrer comme un phénomène authentiquement sensible et immédiatement suprasensible. Il faut en conclure que, dans l'expérience de la Révélation comme dans l'amour et la parole qui en sont les images, «la différence entre "immanence" et "transcendance" s'estompe ${ }^{26} »$. Cette situation herméneutique s'explique, en dernière instance, par le fait que nous avons affaire à la consignation écrite d'un dialogue : la parole de l'homme langage par lequel nous entrons en rapport avec Dieu - se trouve ici immédiatement dans la parole de Dieu, c'est-à-dire dans le texte sacré. Le caractère métaphorique du Cantique ne fait donc pas signe vers un sens transcendant à la lettre du texte, mais il est l'indice de sa dimension dialogique. L'accès au sens du texte suppose, contre toute allégorèse, que l'on reconnaisse l'autonomie de la métaphore.

La Conférence sur l'éthique semble adopter une position plus négative à l'encontre de la métaphore. Wittgenstein remarque que les énoncés éthiques ou religieux ne se distinguent pas par le choix d'un vocabulaire spécifique, mais par le fait que nous y conférons un caractère absolu à des termes qui, dans le langage ordinaire, n'expriment qu'une valeur relative ; nous parlons par exemple du « bien absolu », de la « conduite absolument correcte », alors que le bon et le correct, appliqués à des faits du monde, ne peuvent avoir de valeur que relative (« un bon joueur de tennis », « la route correcte pour aller à Grantchester »). Je déclare ainsi éprouver un sentiment de sécurité absolue,

23. Ibid., p. 221-222 (trad. française p. 281).

24. Ibid., p. 224 (trad. française p. 284, traduction modifiée).

25. Ibid., p. 221 (trad. française p. 281). Sur cette question, voir Myriam Bienenstock, «L'amour dans le Cantique des Cantiques: "juste une comparaison” (Gleichnis)?», Les Études philosophiques, 2019/2, n¹92, p. 299-314.

26. F. Rosenzweig, Der Stern der Erlösung, op. cit., p. 222 (trad. française p. 282, traduction modifiée). 
quoi qu'il puisse m'arriver; je m'étonne non pas de ce que le monde présente telle ou telle qualité, mais de son existence en général. À l'évidence, ces propositions font un usage irrégulier des termes qu'elles emploient : il n'y a de sens à s'étonner que d'une chose dont je pourrais concevoir qu'elle ne se produise pas ; puisque je ne peux pas imaginer que le monde n'existe pas, je ne devrais pas non plus pouvoir m'étonner de son existence. L'expression de mon étonnement est un non-sens ${ }^{27}$.

C'est toutefois le caractère métaphorique de cet usage dépourvu de sens qui lui assure un semblant de légitimité. Parce qu'il existe une analogie entre certaines choses dont nous parlons en termes relatifs - la valeur d'un bijou et d'autres auxquelles nous voulons conférer un caractère absolu - la valeur d'une vie humaine -, nous sommes tentés de dire que nous procédons simplement au déplacement analogique d'un terme de valeur ; c'est en étant transféré d'un objet à un autre qui lui est analogue que le mot passe à la puissance absolue qui est la sienne dans l'énoncé éthique. Il semble que tel soit le fondement de nos expressions portant sur Dieu. Toutefois, il n'y a là qu'une apparence qui se dissipe dès que l'on examine la grammaire du mot de métaphore (simile) :

Mais une métaphore doit être la métaphore de quelque chose. Et si je puis décrire un fait au moyen d'une métaphore, il faut également que je puisse laisser tomber cette métaphore et décrire les faits sans recourir à elle. Or, dans notre cas, dès que nous essayons d'écarter la métaphore et de simplement décrire les faits qui se trouvent derrière elle, nous découvrons qu'il n'existe aucun fait de ce genre. C'est ainsi que ce qui nous était d'abord apparu comme une métaphore semble n'être plus qu'un simple non-sens ${ }^{28}$.

L'interprétation en termes de métaphore est donc le masque ou l'alibi du non-sens, qu'elle introduit en contrebande dans notre langage. La situation est analogue à celle qu'évoquent les Leçons sur la croyance religieuse: l'absence d'une réalité que l'on pourrait montrer indépendamment de la métaphore fonde certes le caractère irremplaçable des images auxquelles le langage religieux a recours, mais neutralise également la catégorie de métaphore, puisque la structure de renvoi qui caractérise cette dernière tourne ici à vide. L'autonomie de la métaphore signifie qu'elle est moins que métaphore, simple non-sens résultant d'un excès illégitime du sens. L'image

27. Comme le rappelle un résumé en allemand de la Conférence dans L. Wittgenstein, Ludwig Wittgenstein und der Wiener Kreis, op. cit., p. 93 : «Erstaunen über die Tatsache der Welt. Jeder Versuch, es auszudrücken, führt zu Unsinn » («L'étonnement devant le fait du monde. Toute tentative pour l'exprimer conduit au non-sens »). Dans le même volume, une réflexion souligne la proximité de Wittgenstein avec Kierkegaard, mais aussi avec Heidegger, dans la conviction que notre tendance à aller nous heurter à la limite du langage fait signe vers quelque chose : "Dieses Anrennen gegen die Grenze der Sprache ist die Ethik. [...] Aber die Tendenz, das Anrennen, deutet auf etwas hin »(Ibidem, p. 68-69). Voir Wittgenstein et le cercle de Vienne, op. cit., p. 65 et p. 38-39.

28. L. Wittgenstein, « Conférence sur l'éthique », op. cit., p. 17. 
linguistique en tant que telle ne constitue d'ailleurs pas le fond du problème : celui-ci tient à ce que nous souhaitons conférer à une certaine expérience une valeur absolue, là où, selon la distinction posée par le Tractatus (6.41), aucun fait du monde ne saurait posséder une telle valeur ${ }^{29}$.

\section{III}

Parvenu à ce point, Wittgenstein décide toutefois d'aller à la rencontre du paradoxe que constitue la valeur absolue d'une expérience, comme si le fait de rejeter l'expression métaphorique du côté du non-sens ne suffisait pas à résoudre la question qu'elle soulève. Wittgenstein s'appuie alors sur la notion de miracle, implicitement présente depuis le début de la conférence : s'étonner de l'existence du monde, c'est voir le monde comme un miracle ; le nom allemand du miracle, das Wunder, résonne dans l'expression anglaise de l'étonnement (I wonder). Est-il possible de mettre ce miracle en mots ? Pour Wittgenstein, il est certain que le miraculeux ne peut pas être exprimé dans le langage, sauf à produire des non-sens qui sont tels par essence, et non parce que nous n'aurions pas encore trouvé une manière de les analyser qui leur donnât un sens. La conclusion de la Conférence confirme que nos tentatives pour exprimer le miraculeux témoignent d'une tendance inextirpable, mais sans espoir, à aller buter contre les limites de notre langage : "This running against the walls of our cage is perfectly, absolutely hopeless $^{30} \gg$.

Une remarque contemporaine de la Conférence, rapportée par Waismann, invite toutefois à nuancer le caractère sans appel de ce propos :

La parole, dans la religion, n'est pas non plus métaphore ; sinon on devrait aussi pouvoir le dire en prose. Se heurter à la limite du langage ? Le langage n'est pas une cage ${ }^{31}$.

L'impropriété de la métaphore est ici associée à une critique de la métaphore de la cage. Voir le langage comme une cage, c'est le traiter comme un moyen d'expression pouvant être mis en échec par certaines expériences « ineffables ». D'après l'interprétation « résolue » du Tractatus, qui inspire dans une certaine mesure nos analyses, Wittgenstein ne

\footnotetext{
29. L. Wittgenstein, Tractatus logico-philosophicus, op. cit., p. 109 : « Dans le monde, tout est comme il est, et tout arrive comme il arrive ; il n'y a en lui aucune valeur - et s'il y en avait une elle serait sans valeur $»$.

30. L. Wittgenstein, « Conférence sur l'éthique », op. cit., p. 19.

31. L. Wittgenstein, Wittgenstein und der Wiener Kreis, op. cit., p. 117 : «Die Reden der Religion sind auch kein Gleichnis; denn sonst müßte man es auch in Prosa sagen können. Anrennen gegen die Grenze der Sprache ? Die Sprache ist ja kein Käfig » (Wittgenstein et le cercle de Vienne, p. 91-92, traduction modifiée).
} 
chercherait pas à montrer les limites du langage, mais justement à dénoncer l'illusion qui nous fait croire que c'est contre elles que nous venons buter ${ }^{32}$. C'est pourquoi la Conférence sur l'éthique suggère une autre distinction : «Or je suis tenté de dire que l'expression correcte, dans le langage, pour le miracle de l'existence du monde, bien qu'elle ne soit pas une proposition dans le langage, est l'existence même du langage $»^{33}$. Il ne pourrait donc $\mathrm{y}$ avoir d'expression du miraculeux au moyen du langage (by means of language), mais seulement par l'existence du langage (by the existence of language). Cette tournure nous frappe par son caractère irrégulier: quel sens y a-t-il à dire qu'une chose est exprimée par l'existence d'une autre, puisque l'existence n'est pas un moyen d'expression ? Cette formule suggère que le langage dont parle ici Wittgenstein n'est plus, lui non plus, à considérer comme un moyen d'expression entretenant une relation d'image avec les faits du monde. L' " existence du langage» est donc autre chose que ce que le Tractatus appelait «l'ensemble des propositions » ${ }^{34}$, le corpus des énoncés dont on peut dire s'ils ont ou non un sens.

Que peut bien vouloir dire que le miraculeux est exprimé par l'existence du langage? Un retour à L'Étoile de la Rédemption mettra peut-être en lumière ce propos mystérieux. La proposition de Wittgenstein offre toutes les apparences d'un théologoumène : si l'existence même du langage est expression du miracle, ce pourrait être parce que nous la devons à un don de Dieu ; elle témoignerait de la relation miraculeuse qui unit l'homme à Dieu, relation que Rosenzweig appelle la Révélation et qui se fonde elle-même dans le miracle de la Création. Or L'Étoile de la Rédemption s'efforce d'ôter à ces contenus théologiques toute dimension mythique. Le don divin de la parole, « cadeau de noces » fait par le Créateur à l'humanité naissante ${ }^{35}$, ne répond pas plus à l'insondable question de l'origine du langage qu'il ne décrit ou prescrit un certain usage de cette parole. Dépourvu de signification empirique, il dit seulement que le sens véritable de la parole est atteint dans l'événement de la Révélation, c'est-à-dire dans le dialogue qui transforme l'ipséité muette en une subjectivité douée d'âme, ouverte à autrui. C'est à ce titre que la Révélation, comme la Rédemption espérée et promise après elle, est un miracle du langage (Sprachwunder ${ }^{36}$ ). De même, pour Wittgenstein, l'existence du langage n'a pas besoin d'attester son origine divine ou d'offrir la description d'un fait empirique pour posséder la valeur d'une expression miraculeuse, sur laquelle il nous reste à faire la lumière.

32. Voir James Conant, "What 'Ethics' in the Tractatus Is Not», in Religion and Wittgenstein's Legacy, op. cit., p. 39-88, ici p. 53 : «The illusion that the Tractatus seeks to explode, above all, is that we can run up against the limits of language ».

33. L. Wittgenstein, "Conférence sur l'éthique », op. cit., p. 18 (traduction modifiée).

34. L. Wittgenstein, Tractatus logico-philosophicus, 4.001, op. cit., p. 50 (traduction modifiée).

35. F. Rosenzweig, Der Stern der Erlösung, op. cit., p. 122 (trad. française p. 161).

36. Ibid., p. 213 (trad. française p. 270). 
Cette démythologisation de l'existence du langage suppose une réévaluation du concept de miracle, que Wittgenstein et Rosenzweig se refusent tous deux à considérer comme un événement extraordinaire venant rompre l'ordre naturel ${ }^{37}$. Dans L'Étoile de la Rédemption, le caractère miraculeux de l'événement consiste en ce qu'il a été prédit, ce qui fait de lui un signe de la divine Providence ${ }^{38}$. En tant que signe, le miracle repose sur une structure de renvoi qui se déploie dans le temps, de la prédiction à l'accomplissement, et non plus, synchroniquement, du signifiant au signifié. Pour Wittgenstein non plus, le miraculeux n'est pas l'extraordinaire ou l'inouï. Le miracle, par exemple la transformation de l'eau en vin, est un geste qui a valeur de symbole de l'esprit dans lequel il a été accompli ; c'est cet esprit lui-même qui est merveilleux ou miraculeux (wunderbar) et qui confère au geste sa signification. Pour qui veut comprendre les miracles du Christ, le miracle doit devenir lui-même une forme de langage : « Pour nous parler, le miracle doit être compris comme geste, comme expression. » Une condition spirituelle doit alors être partagée par l'acte et par son interprète : non seulement « ce n'est que s'il fait ce qu'il fait dans un esprit miraculeux qu'il est un miracle », mais aussi « il me faut déjà lire tout cela dans l'esprit qui convient [in dem richtigen Geiste] pour y sentir le miracle ${ }^{39} »$. Une note ultérieure confirmera que, si le miracle est en un certain sens " un geste de Dieu », se traduisant dans le monde par un événement qui est lui-même « un geste symbolique, un geste de la nature », la croyance en ce miracle dépend de ma capacité à être impressionné. Croire en le miracle signifie ne pas pouvoir voir l'événement sans voir aussi autre chose, c'est-à-dire sans penser ou avoir le sentiment qu'il y a là une action de la divine providence ${ }^{40}$. Le sens du miracle n'est donc pas caché derrière le symbole, mais tributaire de la manière de lire qui est la mienne. Le concept de miracle permet à Wittgenstein et Rosenzweig d'esquisser une autre théorie du signe, qui libère celui-ci de l'exigence descriptive à l'égard du monde.

Le propos de la Conférence sur l'éthique sur l'expression du miracle par l'existence du langage pourrait alors se comprendre de la façon suivante :

37. Sur l'interprétation du miracle chez Rosenzweig, voir Paul Mendes-Flohr, « Rosenzweig's Concept of Miracle », in J. Mattern, G. Motzkin, S. Sandbank (éd.), Jüdisches Denken in einer Welt ohne Gott. Festschrift für Stéphane Mosès, Berlin, Verlag Vorwerk 8, 2000, p. 53-66; chez Wittgenstein, voir tout récemment Hent de Vries, Miracles et métaphysique, Paris, Presses Universitaires de France, 2019, Cinquième leçon, p. 299-355.

38. F. Rosenzweig, Der Stern der Erlösung, op. cit., p. 104-105: pour la conscience des temps bibliques, à la différence de la conscience moderne, le caractère miraculeux du miracle « ne repose pas sur le fait qu'il dévie du cours de la nature défini à l'avance par des lois, mais sur le fait qu'il a été prédit. Le miracle est essentiellement "signe" " (trad. française p. 141, traduction modifiée).

39. L. Wittgenstein, Carnets de Cambridge et de Skjolden, 1930-1932, 1936-1937, traduits de l'allemand et présentés par Jean-Pierre Cometti, Paris, Presses Universitaires de France, 1999, p. 64-65.

40. L. Wittgenstein, Remarques mêlées, édité par G.H. von Wright en collaboration avec Heikki Nyman, trad. Gérard Granel, présentation et notes par Jean-Pierre Cometti, Paris, GFFlammarion, 2002, p. 107-108. 
s'il demeure vrai que l'existence du langage se réalise dans des propositions, ce qui est véritablement miraculeux n'est pas le contenu de ce que l'on dit, mais bien plutôt l'esprit dans lequel cela est dit. C'est pourquoi les énoncés du langage religieux peuvent bien neutraliser la fonction représentative du langage - ils ne disent rien sur le monde : à qui voudrait les comprendre comme des énoncés descriptifs, ils opposent un non-sens irréductible -, sans perdre, pour autant, leur fonction expressive. Tenir un propos éthique ou religieux n'est pas décrire un fait du monde ou hors du monde, c'est faire un geste qui manifeste un certain esprit. En ce sens, l'expérience de voir le monde comme un miracle illustre ce que le Tractatus (6.43) décrivait comme l'effet du bon ou du mauvais vouloir, qui ne change rien aux faits du monde, mais en modifie les frontières. Le monde " doit alors devenir par là totalement autre », « diminuer ou croître dans son ensemble ${ }^{41} »$, « comme par l'acquisition ou la perte d'un sens ${ }^{42} »$, ajoutent les Carnets de 1916.

Telle est la raison fondamentale pour laquelle la notion de métaphore n'est pas pertinente. Si les propos religieux étaient tenus à titre seulement métaphorique, l'on pourrait les reformuler en éliminant la métaphore, ce qui reviendrait à les traiter comme des propos portant sur le monde. La fin de la Conférence sur l'éthique écarte résolument cette possibilité : nous rejetterions toute reformulation de ce type précisément en raison de son caractère sensé (on the ground of its significance ${ }^{43}$ ). Par là, Wittgenstein ne rappelle pas seulement que le non-sens des énoncés religieux leur est irréductible en ce sens qu'on ne saurait leur conférer un contenu descriptif. Il montre aussi qu'une reformulation non métaphorique irait à l'encontre de ce que cherche précisément à effectuer le langage religieux : une transformation du monde. La parole dans la religion n'est pas simple métaphore, déplacement du sens vers son excès, au risque de se heurter à ses limites ; elle ne vise pas l'expression de ce qui est par les moyens du langage, mais sa transformation effective par l'existence du langage. S'offre-t-elle encore, à ce titre, à notre compréhension ? Reprenant la distinction de la fin du Tractatus, nous pourrions dire que les phrases du langage religieux ne peuvent peut-être pas être comprises, mais que celui qui les dit peut être compris, à condition que l'on sache « lire le tout dans l'esprit correct ${ }^{44}$, lui qui seul permet de « voir correctement le monde ${ }^{45} »$.

Pour faire droit à la manière de signifier des énoncés religieux, une nouvelle conception du signe linguistique et de sa nature d'image est donc requise, qui conduit les deux auteurs, selon l'expression de Hent de Vries, à « revisiter l'archive de l'idiome religieux ainsi que la fonction critique qui

41. L. Wittgenstein, Tractatus logico-philosophicus, op. cit., p. 110.

42. L. Wittgenstein, Carnets 1914-1916, trad. Gilles-Gaston Granger, Paris, Gallimard/Tel, 2005 , p. 140.

43. L. Wittgenstein, «Conférence sur l'éthique », op. cit., p. 18.

44. L. Wittgenstein, Carnets de Cambridge et de Skjolden, op. cit., p. 65.

45. L. Wittgenstein, Tractatus logico-philosophicus, op. cit., p. 112. 
peut toujours ou à nouveau lui être assignée ${ }^{46}$. Les apories de la métaphore conduisent en effet la pensée à emprunter le détour du miracle : à la structure de déplacement de la métaphore, les deux auteurs substituent une autre structure de renvoi, de l'événement à sa prédiction (Rosenzweig) et de l'acte à l'esprit qui l'inspire (Wittgenstein). Cette différence reflète l'écart entre les miracles qui leur servent de modèle : si Rosenzweig fait référence à l'action providentielle de Dieu et à ce miracle par excellence qu'est la Révélation, Wittgenstein a en tête les miracles du Nouveau Testament, qui ne prennent sens qu'à être rapportés à l'instance subjective qui en est l'auteur, le Christ ${ }^{47}$. Plus profondément encore, les deux auteurs aboutissent, en dépit d'un cheminement similaire, à des conceptions différentes de ce langage dont l'existence même est expression du miracle. Parce qu'il est le signe d'un événement qui est une rencontre, le langage s'accomplit, chez Rosenzweig, comme dialogue; dès lors, c'est dans la relation au prochain que s'opère la transformation du monde, dont le sens est d'anticiper la Rédemption en faisant croître le Royaume dans le monde ${ }^{48}$. En tant que, pour Wittgenstein, le langage religieux est le symbole d'un certain esprit que le croyant veut adopter ou qu'il sait être déjà le sien, la transformation ne s'opère pas tant dans la relation à l'autre que dans l'aspiration à une vie plus parfaite ; le sens du monde change sous l'effet de l'accord que je noue avec lui, qui est aussi l'harmonie « avec cette volonté étrangère dont je parais dépendre ${ }^{49} »$. Pardelà la métaphore et le miracle, c'est donc le sens d'une conception éthique de l'existence qui est en jeu ici, ainsi que le sens de cette transformation du monde à laquelle doit avoir part la langue des hommes.

46. Hent de Vries, Miracles et métaphysique, op. cit., p. 352.

47. Par là, Wittgenstein se rapproche peut-être de la fonction dévolue aux miracles par Kierkegaard, notamment dans L'Exercice en christianisme (trad. Vincent Delecroix, Paris, Éditions du Félin, 2006) : le miracle n'est jamais une preuve de la divinité du Christ, mais un signe de contradiction destiné à provoquer le scandale. Dans l'apparition du Christ, « il n'y avait directement rien à voir qu'un homme de rien, qui par signes et miracles et en affirmant qu'il était Dieu, sans relâche, instaurait la possibilité du scandale » (p. 107). Pour celui qui s'est rendu contemporain du Christ, le miracle est un signe offensant qui, en soumettant l'existence à une tension particulièrement vive, prépare à la foi (p. 80), rend l'esprit attentif en l'obligeant à se heurter à la contradiction (p. 179).

48. Voir le chapitre consacré à la Rédemption dans F. Rosenzweig, Der Stern der Erlösung, op. cit., p. 229-282 (trad. française p. 290-356).

49. L. Wittgenstein, Carnets 1914-1916, op. cit., p. 142. 